\title{
Harnblasenkarzinom
}

\section{Spiegelung verfälscht die Zytologie}

\begin{abstract}
Die Uringewinnung per Zystoskop bei Makrohämaturie kann das Testergebnis bei Verdacht auf ein Harnblasenkarzinom verfälschen, wie eine niederländische Studie bestätigt. Die Anwendung des Tests auf Cytokeratin 20 (CK 20) bei Verwendung von Spontanurin kann die Sensitivität erhöhen, ohne die Spezifität zu schmälern.

Für die Studie wurden 160 Urinproben von 80 Patienten mit Makrohämaturie im Alter von durchschnittlich 65,8 Jahren ausgewertet. Je zur Hälfte waren die Proben spontan oder während der Blasenspiegelung gewonnen worden. Bei 37 Patienten $(46,3 \%)$ wurde wegen verdächtiger Läsionen eine transurethrale Resektion oder eine endoskopische Untersuchung der Harnleiter vorgenommen. Bei den meisten Patienten (91,2\%) wurde eine CT-Urografie vorgenommen, nur bei sechs Patienten eine sonografische Untersuchung der Nieren und der Blase.
\end{abstract}

\section{kurz notiert}

Forschungsstandort Deutschland

Biomarker für Progression identifiziert +++ Wissenschaftler aus Freiburg stellten fest, dass die Tumorzellen eines nichtkleinzelligen Lungenkarzinoms deutlich mehr Protein MTSS1 produzieren als nicht entartete Zellen. Besonders interessant war, dass Tumoren in frühen Stadien mehr MTSS1 produzierten als solche, bei denen bereits ein fortgeschrittenes Tumorstadium vorlag. Die Forscher vermuten, dass die Bestimmung des Proteins sogar eine bevorstehende Metastasierung erkennen lassen könnte [Kayser G et al. Br J Cancer. 2015;112(5): 866-73].

CD95 macht Tumor aggressiv +++

Pankreaskrebszellen tragen deutlich mehr CD95Proteine auf ihrer Oberfläche als gesunde Zellen der Bauchspeicheldrüsen, und Krebszellen, die besonders viel CD95 bilden, zeigen die deutlichsten Merkmale in Richtung Malignität. Krebszellen aus Metastasen tragen darüber hinaus mehr CD95 als Zellen des Primärtumors. Das beobachteten Wissenschaftler des Deutschen Krebsforschungszentrums in Heidelberg. Außerdem wiesen sie nach, dass CD95 eine ursächliche Rolle für die Aggressivität eines Pankreaskarzinoms spielt [Teodorczyk M et al. Cell Death Differ. 2015; 22(7):1192-202].
Bei 26 Patienten (32,5\%) gab es durch die Bildgebung einen Verdacht auf ein Malignom. 30 Patienten (37,5\%) hatten ein histologisch nachgewiesenes Harnblasenkarzinom, das bei vier Patienten muskelinvasiv war. Es handelte sich bei 17 Patienten (21,3\%) um ein Hochrisikomalignom, bei zwölf Patienten (15\%) um ein intermediäres Karzinom und nur bei einem Patienten um ein Niedrigrisikokarzinom.

Den Berechnungen zufolge hatte die Routinezytologie mit Spontanurin eine signifikant höhere Spezifität als mit während der Spiegelung gesammeltem Urin (94 vs. $72 \%$ ). Die Sensitivität der Zytologie - also mit richtig positivem Testergebnis - war in beiden Fällen deutlich niedriger. Die Werte unterschieden sich aber nicht signifikant (40 vs. $53 \%$ ). Durch die Anwendung der CK-20-Analyse ließ sich die Sensitivität bei der Untersuchung des Spontanurins allerdings deutlich erhö-



Spontanurin ist für die Zytologie besser geeignet als Urin aus der Blasenspiegelung.

hen, sodass der Unterschied zur Sensitivität in der Vergleichsgruppe mit alleiniger Zytologie signifikant wurde (67 vs. $40 \% ; \mathrm{p}=0,03)$. Keinen signifikanten Unterschied brachte der Test jedoch bei der Verwendung von Urin, der während der Spiegelung gewonnen wurde (67 vs. $53 \%$; $\mathrm{p}=0,30)$.

Peter Leiner

Wegelin $\mathrm{O}$ et al. The effects of instrumentation on urine cytology and CK-20 analysis for the detection of bladder cancer. Urology. 2015; Jul 9 (Epub ahead of print).

\section{Metastasiertes Pankreaskarzinom nab-Paclitaxel auch langfristig erfolgreich}

Die Langzeitdaten der MPACT-Studie bestätigen, dass die Addition von nab-Paclitaxel zu Gemcitabin den Therapieerfolg beim metastasierten Pankreaskarzinom verbessern kann. In der Studie hatten 861 Patienten mit metastasiertem Pankreaskarzinom entweder Gemcitabin allein $\left(1.000 \mathrm{mg} / \mathrm{m}^{2}\right.$ an den Tagen 1,8 und 15) oder zusätzlich vor jeder GemcitabinGabe eine intravenöse Infusion mit 125 $\mathrm{mg} / \mathrm{m}^{2}$ nab-Paclitaxel erhalten.

In der nun publizierten Post-hoc-Analyse wurden auch Patienten erfasst, die länger als 24 Monate lebten. Dies traf auf $10 \%$ der Patienten in der Kombinationsgruppe und auf $5 \%$ in der GemcitabinGruppe zu. Unter der Kombinationtherapie lebten manche Patienten auch bis zu 42 Monate, während dies im GemcitabinArm nicht der Fall war.

Das mittlere Gesamtüberleben im Kombinationsarm war signifikant länger (8,7 vs. 6,6 Monate; Hazard Ratio [HR] $0,72 ; \mathrm{p}<0,001)$. In Subgruppen von Pati- enten fand sich aber ein günstiger Effekt der Kombinationstherapie auf das Gesamtüberleben: Bei CA19-9-Spiegeln, die höher als der Durchschnitt waren, lag die Hazard Ratio bei 0,612 (p < 0,001) und bei einem Neutrophilen-Lymphozyten-Quotienten $>5$ bei $0,81(p<0,079)$.

Allerdings ging die Kombinationstherapie auch mit stärkeren Nebenwirkungen einher: So traten Neutropenie, Leukopenie, Fatigue, periphere Neuropathie und Diarrhö häufiger auf, nicht aber Anämie oder Thrombozytopenie gleich welchen Grades. Die Zahl der Patienten, die eine Nebenwirkung vom Schweregrad $\geq 3$ erlitten, lag bei 326 (77\%) in der nabPaclitaxel/Gemcitabin-Gruppe und bei 205 (51\%) im Gemcitabin-Arm.

Christina Berndt

Goldstein D et al. Nab-Paclitaxel Plus Gemcitabine for Metastatic Pancreatic Cancer: Long-Term Survival From a Phase III Trial. J Natl Cancer Inst. 2015; 107:dju413. 